Symptom centrality and infrequency of endorsement identify adolescent depression symptoms more strongly associated with life satisfaction

\author{
Michael C. Mullarkey ${ }^{1}$, Igor Marchetti ${ }^{2}$, Karen Bluth $^{3}$, Caryn L. Carlson ${ }^{4}$, Jason Shumake $^{4}, \&$ \\ Christopher G. Beevers ${ }^{4}$ \\ ${ }^{1}$ Stony Brook University \\ ${ }^{2}$ University of Trieste \\ ${ }^{3}$ University of North Carolina-Chapel Hill \\ ${ }^{4}$ University of Texas at Austin
}

Corresponding Author: Michael C. Mullarkey

E-mail:michael.mullarkey@stonybrook.edu

Address: Stony Brook University Psychology B Building, Room 340

Stony Brook, NY 11794-2500

Funding: This research did not receive any specific grant from funding agencies in the public, commercial, or not-for-profit sectors.

Declarations of Interest: The funders have/had no role in study design, data collection and analysis, decision to publish or preparation of the manuscript. Unrelated to the present study, MCM is under contract with New Harbinger Publications to co-author a therapeutic workbook for adolescents. CGB reports receiving grant support from the National Institute of Health and compensation for his work as journal editor for Sage Publications Inc. The authors report no other financial or non-financial conflicts. 


\begin{abstract}
Although depression symptoms are often treated as interchangeable, some symptoms may relate to adolescent life satisfaction more strongly than others. To assess this premise, we first conducted a network analysis on the Mood and Feelings Questionnaire (MFQ) in a large ( $N=$ 1,059 ), cross-sectional sample of community adolescents (age $M=14.72 \pm 1.79$ ). The most central symptoms of adolescent depression, as indexed by strength, were self-hatred, loneliness, sadness, and worthlessness while the least frequently endorsed symptoms were self-hatred, anhedonia, feeling like a bad person, and feeling unloved. Moreover, the more central a depression symptom was in the network (i.e., higher strength), the more variance it shared with life satisfaction $(r=0.59,95 \% \mathrm{Cl}: 0.27,0.76)$. How frequently a symptom was endorsed was negatively associated with the variance symptoms shared with life satisfaction ( $r$ $=-0.48,95 \% \mathrm{Cl}:-0.63,-0.21)$. Cross-validated, prediction focused models found central symptoms were expected to predict more out of fold variance in life satisfaction than peripheral symptoms and frequently endorsed symptoms, but not the least frequently endorsed symptoms. These findings show certain depression symptoms may be more strongly associated with life satisfaction in adolescence and these symptoms can be identified by multiple symptom-level metrics. Limitations include use of cross-sectional data and utilizing a community sample. Better understanding which symptoms of depression share more variance with important outcomes like life satisfaction could help us develop a more fine-grained understanding of adolescent depression.
\end{abstract}

Keywords: adolescent depression, symptom-level, network analysis, life satisfaction 


\section{Symptom centrality and infrequency of endorsement identify adolescent depression symptoms more strongly associated with life satisfaction}

Depression symptoms are common in adolescents-approximately $18.6 \%$ experience clinical levels (Lu, 2019) and more than $20 \%$ may experience subclinical levels of depression (Bertha \& Balázs, 2013). However, depression is far from a homogenous syndrome (Fried, 2017). For example, $48.6 \%$ of 3,703 adults enrolled in a depression treatment trial had a unique pattern of symptoms that no one else endorsed (Fried \& Nesse, 2015).

This heterogeneity would not be consequential if all symptoms were equally important, but depressive symptoms are differentially linked to cognitive risk factors, cognitive biases, and psychosocial functioning in adults (Beevers et al., 2018; Fried \& Nesse, 2014; Marchetti et al., 2016, 2018). For instance, some symptoms (sadness) shared as much as $20.7 \%$ of the variance with functioning outcomes, while others (hypersomnia) shared as little as $0.7 \%$. This pattern of findings appears to extend to adolescents, as anhedonia is associated with suicidal ideation in adolescents while irritability is not (Gabbay et al., 2015). Ignoring the intrinsic heterogeneity of depression may mask important associations between individual depression symptoms and non-symptom outcomes in adolescents, such as life satisfaction (Fried, 2015; Zadow et al., 2017).

The network theory of psychopathology provides one framework for identifying symptoms that might be important for a variety of outcomes. In this model, symptoms are not outcomes of an underlying disease. Instead, symptoms and the associations between them are the disease (McNally, 2016), in that symptoms (i.e., nodes) may have reciprocal links (i.e., edges) with one another. Some edges may be stronger than others, and this may lead to a network structure where some symptoms are more central, or important, to the network than others. Testing whether these more central symptoms are actually more important to depression 
relevant outcomes is crucial; assuming importance based on centrality in a network model alone is a problematic inference (Fried et al., 2018).

A previous network analysis in a large, diverse sample of adolescents has identified selfhatred, loneliness, sadness, and pessimism as the most central symptoms of adolescent depression (Mullarkey et al., 2018). However, that study did not link central symptoms to other depression-relevant outcomes. Further, it is essential to assess whether initial findings converge with results in different samples using other measures (Vazire, 2018). None of the indices for the replicability of central symptoms can be applied when utilizing a different measure (Borsboom et al., 2017), but we can descriptively assess whether the symptoms identified as most central in previous analyses are found to be more central in this new sample. We can also utilize newly developed Bayesian methods to directly assess the replicability of the estimated network within this sample (Jones et al., 2020). Other domains of psychopathology in adolescence have similar central symptoms when networks are estimated on different measures (McCuish et al., 2019), but whether this is the case in adolescent depression remains an open, empirical question. Among all the important outcomes associated with adolescent depression, life satisfaction plays a major role (Proctor et al., 2009). For instance, a representative, population based study of adolescents shows life satisfaction is related to positive social and health outcomes (Gadermann et al., 2016). Moreover, life satisfaction is related to but distinct from the absence of depression or other psychopathology (McKnight \& Kashdan, 2009) and experiencing symptoms such as sadness are often orthogonal to perceiving one's life as positive (Crowe et al., 2018) and satisfying (Schimmack, 2008).

Still, most prior work linking life satisfaction and depression is based on sum scores of symptoms, while some symptoms of adolescent depression may be more strongly associated with life satisfaction than others. Importantly, network centrality is not the only symptom-level metric that could identify potentially more relevant symptoms. For example, changes in symptoms that were less frequently endorsed were more predictive of overall treatment 
response to social anxiety disorder than changes in symptoms identified as more central (Rodebaugh et al., 2018). Thus, although network analysis is a promising approach for identifying key symptoms, other approaches should also be investigated to obtain a more complete picture of symptom importance for depression-related outcomes.

Therefore, in the current study, we first identify symptoms that are most central in the depression symptom network and determine how well the central symptoms converge with previous work while using a different measure of depression in a different sample as has been done with other domains of psychopathology in adolescence (McCuish et al., 2019). We expect that self-hatred, loneliness, and sadness (pessimism was not measured in the current study) will be identified as the most central symptoms. Then, we will investigate whether the most central symptoms in the network (i.e., network-centrality approach), as compared to the most peripheral symptoms (i.e., the least central symptoms in the network-centrality approach) and the most/least often endorsed symptoms (symptom endorsement approach), are more strongly associated with life satisfaction in adolescence. The goal of these analyses is to identify which depression symptoms are most strongly associated with life satisfaction in adolescents.

\section{Method}

\section{Participants}

The adolescent (age $M=14.72 \pm 1.79$ years old; range: $11-19$ years; $12-17$ years old = $94.28 \%$ of sample) sample $(n=1,059)$ was racially diverse $(68.08 \%$ White/Caucasian, $16.24 \%$ Black/African-American, 3.49\% Hispanic, 4.34\% Asian/Pacific Islander, 1.04\% Native American, $5.85 \%$ Other, $0.94 \%$ missing) and predominantly female $(64.97 \%$ female, $32.39 \%$ male, $1.60 \%$ transgender/gender-unsure, $1.04 \%$ missing). Socioeconomic status of the sample as indexed by maternal education was also diverse $(5.85 \%$ did not finish high school, $17.37 \%$ high school or 
equivalent, $14.54 \%$ some college courses but no college degree, $32.96 \%$ bachelor's degree, $19.64 \%$ master's degree, $7.65 \%$ lawyer, doctor, or PhD, $8.58 \%$ don't know, $1.98 \%$ missing).

Adolescents were universally invited to participate in this study at one public middle school, one public high school, and one private middle/high school; all samples were located in the Southeastern United States. Dates of recruitment and data collection were October to December of 2015. Our sample size was determined by our consent rate at these schools. More detailed recruitment details can be found in two previous studies evaluating different outcomes with the middle and high school participants (Bluth et al., 2017, 2018).

A total of 1,059 participants opted in to the study (informed consent was obtained from all individual participants included in the study, 58 participants opted out), correctly answered two out of three attention probe questions during the survey (244 participants excluded), and had no missing item-level data on the depression symptom inventory (6 participants excluded). These exclusions were performed to reduce bias due to inattention (Hauser \& Schwarz, 2016) and distorted correlations in the network (Borsboom, 2017). All participants completed the survey during their school day and provided online assent. The study was approved by the IRBs of all relevant institutions.

\section{Measures}

Additional measures, unrelated to the current study's goal, were also administered. For the current analyses, the following two measures were utilized:

\section{Mood and Feelings Questionnaire-Short Form}

Depressive symptoms were evaluated with the Mood and Feelings Questionnaire-Short Form (MFQ; Thapar \& McGuffin, 1998). The MFQ is a 13-item self-report questionnaire, and all items are measured on a 3-point Likert scale, 0 ("Not True") , 1 ("Sometimes"), and 2 ("True") in response to statements about symptoms in the past two weeks (e.g., "I hated myself"). The 
MFQ is used in research and clinical practice with children and adolescents in a variety of settings (Kuo et al., 2005; McKenzie et al., 2011).

\section{Student's Life Satisfaction Scale}

Life satisfaction was evaluated with the Student's Life Satisfaction Scale (Huebner, 1991). The SLSS is a 7-item self-report questionnaire and all items are measured on a 4-point Likert scale ranging from 0 (“Never") to 3 (“Almost always") in response to general statements about one's life satisfaction (e.g., "I have a good life"). The scale has adequate test-retest reliability across one year $(r=0.53)$ and has a moderate negative correlation with depression $(r$ $=-0.39)$ (Huebner et al., 2000). Importantly, the scale has only one latent factor, making it psychometrically appropriate to sum score (Huebner, 1991). The internal consistency in this sample was $\alpha=0.85$.

\section{Statistical Analysis}

\section{Descriptive Statistics}

We first inspected mean, standard deviation, kurtosis, and skewness of all the MFQ items. In line with previous studies (Mullarkey et al., 2018; Rhemtulla et al., 2016), we dichotomized the single, item-level values to signify the absence (i.e., "0" at the MFQ item) or presence (i.e., "1" or "2" at the MFQ item) of depressive symptoms. We applied such an approach, because the optimal way to model networks with trichotomous items is still under debate (Fried et al., 2015).

\section{Network Estimation}

Using binary symptoms, it was possible to estimate network models with the eLasso Ising model (van Borkulo et al., 2014). An Ising model can be conceived as a series of pairwise associations among binary variables, after controlling for all the other associations, while shrinking potentially spurious relationships to 0 by means of eLasso regularization (for a more technical description see van Borkulo et al., 2014). This type of network estimation results in a 
sparser, easier to interpret network. The binary network was fitted using the $R$-package IsingFit 0.3.1 (van Borkulo et al., 2014) and was visualized using R-package qgraph 1.4.3 (Epskamp et al., 2012). In this visualization blue edges denote positive associations, with thicker edges representing stronger associations.

Symptom centrality was operationalized as strength centrality - the sum of the absolute weights of the edge connecting the node to all the other nodes (Valente, 2012). Strength centrality is consistently, statistically more robust than other indices of centrality, and there is evidence that other indices of centrality may be less valid metrics in psychopathology networks (Bringmann et al., 2018).

We tested the stability and accuracy of our network via both a case-dropping bootstrap test for centrality stability and bootstrap confidence intervals for the accuracy of network edges (Epskamp et al., 2017). Centrality and edge-weight stability was operationalized with the correlation stability coefficient (i.e., CS-coefficient). CS-coefficient represents the maximum proportion of cases that can be dropped to still obtain a $95 \%$ probability that the correlation of the ranking between the centrality indicator/edge-weight in the original network and the casesubset network will amount to 0.7 , with CS-coefficients being required to match at least 0.25 , but preferentially above 0.5 (Epskamp et al., 2017). In addition, internode centrality difference test was computed with $95 \%$ non-parametric bootstrap confidence intervals (Cls; 1,000 bootstrap samples). If zero is included in $\mathrm{Cl}$, the two nodes do not differ with respect to that centrality index. All stability and accuracy analyses described above were performed using the $R$-package bootnet 1.0 (Epskamp et al., 2017).

Then, we calculated bootstrapped correlations between strength and both endorsement and standard deviation of symptoms in the full sample network. This test allows us to determine whether symptom centrality indexes are substantially influenced by symptoms' differential variability or mean values (Terluin et al., 2016), and treats centrality as the interdependent statistic it is (i.e., if one symptom becomes more central numerically at least one other symptom 
will have to become less central numerically) rather than as the independent observations they would be assumed to be using a Pearson or Spearman correlation (Mullarkey et al., 2019).

\section{Bayesian Test of Network Replicability}

We also wanted to directly test how well we would expect this network to replicate in new, similar samples. We first created random split-halves of the data (a random 529 participants in one split-half, a random 530 participants in the other split-half), and then computed Bayes Factors for the strength of evidence that the strength of each network edge was the same across split-halves $\left(\mathrm{H}_{0}\right)$ or different across split-halves $\left(\mathrm{H}_{1}\right)$. Direct evidence for $\mathrm{H}_{0}$ allows us to be more confident the network edges would replicate in new, similar samples while accounting for sampling variability (Jones et al., 2020; Williams et al., 2020). The prior for our analysis was an unrestricted model, or a model agnostic to the size of the partial correlations (Marsman \& Wagenmakers, 2017). We considered Bayes Factors $>3$ to be evidence for the null hypothesis, Bayes Factors $<1 / 3$ to be evidence for the alternative hypothesis, and Bayes Factors $<3$ but $>1 / 3$ to be insufficient information to determine whether either hypothesis is more plausible (Jones et al., 2020). We then repeated this split-half process 10 times to ensure that the results were not unduly influenced by indiosyrancies associated with any given split in the sample and reported the average percentage of edges with evidence for replication $\left(\mathrm{H}_{0}\right)$, against replication $\left(\mathrm{H}_{1}\right)$, and insufficient evidence for either model across these 10 iterations.

\section{Relative Importance of Depression Symptoms for Life Satisfaction}

We then examined whether a symptom's network centrality relates to how much variance that symptom shares with life satisfaction. We first estimated the amount of variance each symptom shared with life satisfaction, as indexed by the Students' Life Satisfaction Scale, using relative importance analysis while controlling for age and gender. To perform the relative importance analysis, we used the $R$-package relaimpo 2.2-2 (Grömping, 2006) and specifically utilized the Img metric. This metric calculates the contribution that each variable makes in accounting for the criterion by itself and in combination with the other predictors. In our study, 
the relative importance partitions were computed in such a way that they summed to $100 \%$ (Grömping, 2007). Each individual predictor's relative importance was also bootstrapped 1,000 times to determine, in an exploratory sense, which predictors shared more variance with life satisfaction and the precision of the estimates (Fried \& Nesse, 2014).

Previous analyses have also correlated centrality with different outcomes like treatment response (e.g., Boschloo et al., 2016), but have reported only point estimates and have not reported estimates of uncertainty for these point estimates. This is because the strength centrality statistics for a network are not independent observations, so confidence intervals or $p$ values based on the assumption of independent and identically distributed observations (e.g., from running a standard correlation test) would not be valid. In other words, a correlation between calculated values from two fixed samples does not have the same sampling distribution as a correlation between measured values from two random samples. Moreover, the sampling distribution for centrality measures is non-parametric and typically estimated by bootstrap (Epskamp et al., 2017; Epskamp \& Fried, 2016). Thus, correlating centrality metrics with other outcomes, using standard methods, may not be appropriate.

Therefore, to properly take into account the sampling error in the estimation of the correlation between centrality measures and other outcomes, we generated 1,000 bootstrap replicates of the correlation between strength centrality and variance shared with life satisfaction by sampling from the dataset 1,000 times with replacement. This simulates the distribution of results we would obtain if we collected 1,000 new samples of the same size as our current sample. This approach allows us to more appropriately estimate the uncertainty around how well the centrality of a depression symptom is associated with the amount of variance each depression symptom shares with life satisfaction. We then repeated this bootstrapping procedure with frequency of symptom endorsement. This test allows us to determine whether how often a symptom is endorsed is associated with how much variance that symptom shares 
with life satisfaction. This bootstrapping style of analysis has been used previously to identify correlations between centrality across data sets (Mullarkey et al., 2019).

While this bootstrapping analysis is robust it does not make full use of the sample size of participants, as the correlation being bootstrapped has an $N$ equal to the number of nodes in the network (13) rather than the sample size $(1,059)$. To test for converging evidence while utilizing the full sample, we also assessed the relative ability for central, peripheral, commonly endorsed, and infrequently endorsed symptoms to predict life satisfaction by comparing cross-validated linear models. We used strength centrality to identify the symptoms that were significantly stronger than $50 \%$ or more of other symptoms in the network, as has been done in previous research (Mullarkey et al., 2018). We then created models with an equivalent number of symptoms scoring highest on the other symptom-level metrics - least central/peripheral, commonly endorsed, and infrequently endorsed.

We first fit separate linear regressions with central (worthlessness, self-hatred, sadness, and loneliness), peripheral (fatigue, crying, restless, concentration), commonly endorsed (fatigue, restless, concentration, and sadness), and infrequently endorsed (anhedonia, selfhatred, bad person, unloved) symptoms predicting life satisfaction. We then calculated the out of fold variance $\left(\mathrm{R}^{2}\right.$ pred $)$ by cross-validating each linear model. This type of model prioritizes variance we would expect the models to predict in new samples (See section S1 for further explanation of cross-validation). All data and code used for all analyses can be found on the Open Science Framework (https://osf.io/pf7qu/).

\section{Results}

\section{Descriptive statistics}

Mean, standard deviation, skewness, kurtosis, and frequency of the MFQ symptoms are reported in Table 1. The overall mean and standard deviation (at the item level) of all symptoms, 
or a traditional mean score, is $M=0.58, S D=0.51$ (or $M_{\text {sum score }}=7.50, S D_{\text {sum score }}=6.57$ ). The symptoms of fatigue, restless, concentration, and sadness were the most commonly endorsed (i.e., third tertile), whereas the symptoms of anhedonia, self-hatred, bad person, and unloved were the least commonly endorsed (i.e., first tertile).

Table 1. Mean, standard deviation, minimum, maximum, skewness, and kurtosis, and frequency of the MFQ Symptoms $(n=1,059)$

\begin{tabular}{|c|c|c|c|c|c|c|c|}
\hline Symptoms & MFQ \# & Mean & Std Dev & Min & Max & $\begin{array}{c}\text { \% Absence } \\
\text { ("0") }\end{array}$ & $\begin{array}{l}\text { \% Presence } \\
\text { ("1 or 2") }\end{array}$ \\
\hline Sadness & 1 & 0.78 & 0.69 & 0 & 2 & 37.11 & 62.89 \\
\hline Anhedonia & 2 & 0.39 & 0.60 & 0 & 2 & 66.67 & 33.33 \\
\hline Fatigue & 3 & 0.92 & 0.78 & 0 & 2 & 35.03 & 64.97 \\
\hline Restless & 4 & 0.80 & 0.75 & 0 & 2 & 39.85 & 60.15 \\
\hline No Good & 5 & 0.47 & 0.69 & 0 & 2 & 63.74 & 36.26 \\
\hline Crying & 6 & 0.53 & 0.75 & 0 & 2 & 62.98 & 37.02 \\
\hline Concentration & 7 & 0.85 & 0.75 & 0 & 2 & 36.92 & 63.08 \\
\hline Self-hatred & 8 & 0.37 & 0.67 & 0 & 2 & 73.47 & 26.53 \\
\hline Bad Person & 9 & 0.35 & 0.62 & 0 & 2 & 72.62 & 27.38 \\
\hline Loneliness & 10 & 0.63 & 0.74 & 0 & 2 & 52.97 & 47.03 \\
\hline Feel Unloved & 11 & 0.35 & 0.65 & 0 & 2 & 74.69 & 25.31 \\
\hline $\begin{array}{l}\text { Worse Than } \\
\text { Others }\end{array}$ & 12 & 0.56 & 0.75 & 0 & 2 & 59.77 & 40.23 \\
\hline Did Wrong & 13 & 0.50 & 0.69 & 0 & 2 & 61.10 & 38.90 \\
\hline
\end{tabular}




\section{Network estimation, centrality and edge stability tests, replicability estimate, and intranetwork comparison}

The network of depressive symptoms, as estimated with the Ising model, is shown in Figure 1 (See Section S2 for Item Informativeness). Sadness, worthlessness, loneliness, and self-hatred are displayed toward the middle of the network, while feeling restless, concentration problems, and fatigue were more peripheral. All edges present in the model indicated positive associations between symptoms. Strength and edge-weights had excellent stability, indicating the order of central symptoms and edge-weights would remain largely consistent across samples (See Section S3 for Stability Tests and Significance Tests for Individual EdgeWeights). Our Bayesian tests with 10 sets of random split-halves of the data indicate on average $91.92 \%$ of edges were best explained by the replication model, $7.94 \%$ of edges had insufficient information to be classified in the replication or non-replication model, and $0.14 \%$ of edges (or less than one edge per test) were best explained by the non-replication model. Therefore, we continued with our assessment of network parameters given converging evidence of stability and replicability across these metrics. 


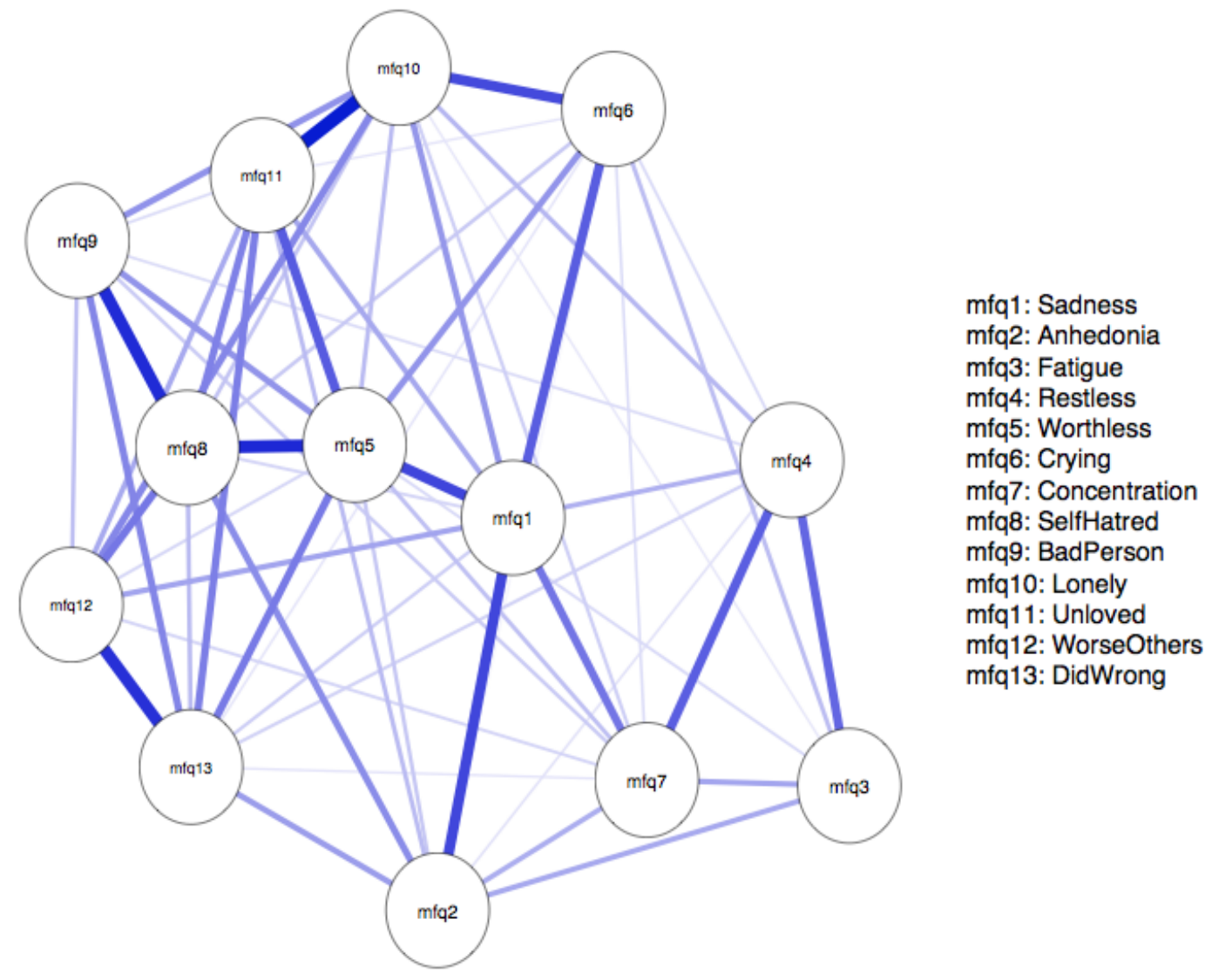

Figure 1 Network of Mood and Feelings Questionnaire depression symptoms in 1,059 adolescents

In terms of strength, feeling worthless, sadness, self-hatred, and loneliness were statistically stronger than $50 \%$ or more of the other symptoms (Figure 2). Thus, these four symptoms appeared to be particularly central in this sample's depression symptom network. In contrast, some symptoms appeared to be peripheral, such as fatigue and feeling restless.

Strength did not significantly correlate with standard deviation $(r=-0.11,95 \% \mathrm{Cl}:-0.35,0.08)$ but did have a moderate negative correlation with symptom endorsement $(r=-0.34,95 \% \mathrm{Cl}$ : 0.53, -0.18, (See Section S4). 


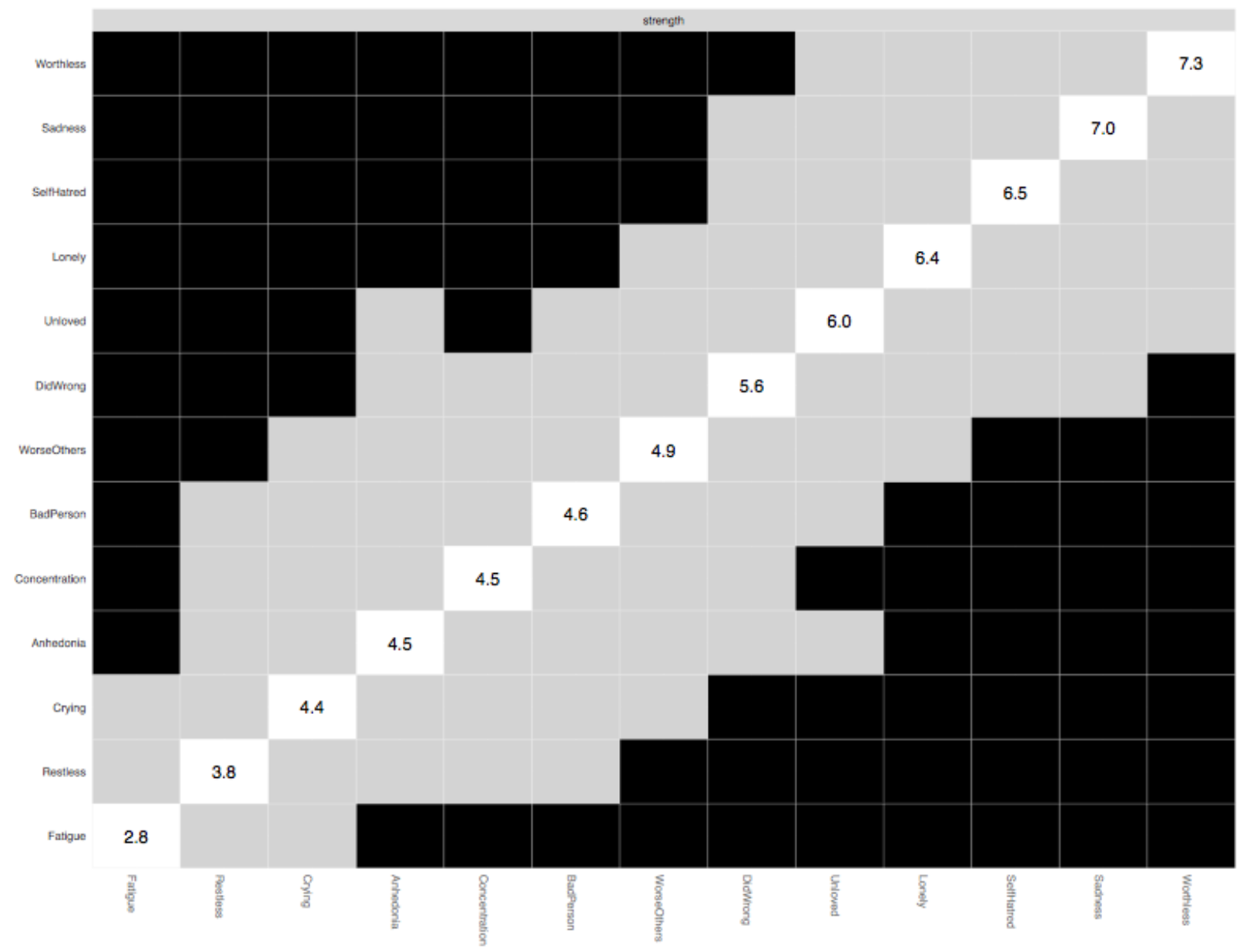

Figure 2 Worthlessness, sadness, self-hatred, and loneliness are the strongest symptoms in this sample's symptom network. Note: Black boxes indicate the symptom is stronger than the other symptom $(p<.05$ following Holm-Bonferroni correction). Gray boxes indicate that symptom strength does not differ between those symptoms

\section{External Validity: Life Satisfaction}

We then conducted a relative importance analysis to determine how much variance the mean of each depressive symptom shares with life satisfaction as indexed by the SLSS. This analysis helps us understand to what extent endorsing different symptoms is associated with a decrease in reported life satisfaction. A linear model with all MFQ symptoms, gender, and age as predictors explains $44.49 \%$ of the variance in life satisfaction. Different symptoms appeared to share widely varying amounts of relative variance with life satisfaction, from as low as $2.9 \%$ 
(feeling restless) to $12.6 \%$ (sadness, Figure 3). These differentials are highlighted by our exploratory bootstrapping analyses, which indicate that 38 out of 80 possible symptom comparisons (47.5\%) differed from one another.

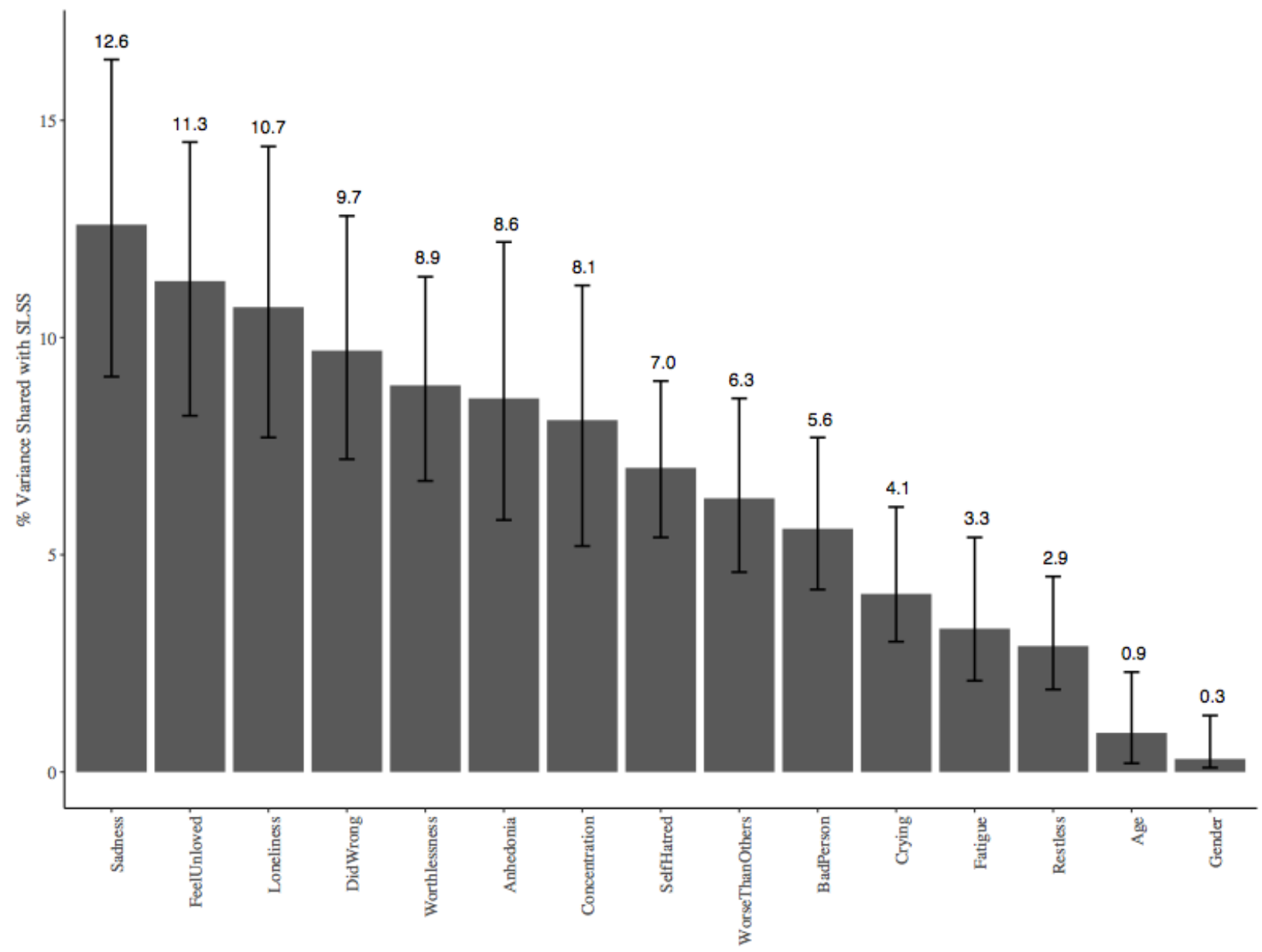

Figure 3 The relative amount of variance individual depressive symptoms share with life satisfaction

Crucially, at a group level across 13 symptoms following bootstrapping, node strength was strongly positively correlated with the amount variance the symptom shares with life satisfaction $(r=0.59,95 \% \mathrm{Cl}: 0.27,0.76)$, indicating that as a symptom becomes more central it becomes more informative about life satisfaction. Further, how often each symptom was endorsed was significantly and negatively correlated with relative importance of the symptom for 
life satisfaction following bootstrapping $(r=-0.48,95 \% \mathrm{Cl}:-0.63,-0.21)$, indicating that as a symptom becomes more commonly endorsed it becomes less informative about life satisfaction. After controlling for symptom endorsement, node strength is still significantly, positively related to variance shared with life satisfaction $(r=0.46,95 \% \mathrm{Cl}: 0.14,0.67)$. When controlling for node strength, infrequency of endorsement was no longer significantly associated with variance associated with life satisfaction $(r=-0.29,95 \% \mathrm{Cl}:-0.48,0.00)$, though the absolute value of its confidence interval still overlapped with node strength's confidence interval.

We then formally compared the cross-validated linear models to utilize the entire sample in assessing whether certain kinds of symptoms better predicted life satisfaction. The central symptoms model predicted $35.55 \%$ of the out of fold variance, the peripheral symptoms model predicted $29.22 \%$ of the variance, and the commonly endorsed model predicted $26.84 \%$ of the variance. The central symptom model had a significantly higher $\mathrm{R}_{\text {pred }}^{2}$ than the peripheral symptoms model $\left(95 \% \mathrm{Cl}\right.$ for $\mathrm{R}_{\text {pred }}^{2}$ difference between the models: $\left.-0.10,-0.02\right)$ and the commonly endorsed model ( $95 \% \mathrm{Cl}$ for $\mathrm{R}^{2}$ pred difference between the models: $\left.-0.12,-0.05\right)$. However, the least frequently endorsed symptoms predicted $37.30 \%$ of the variance, and neither model had a significantly higher $\mathrm{R}_{\text {pred }}^{2}$ than the other $\left(95 \% \mathrm{Cl}\right.$ for $\mathrm{R}_{\text {pred }}^{2}$ difference between the models: $-0.02,0.05)$.

These results retained the same pattern when we compared models containing symptoms uniquely identified by centrality (sadness, loneliness, worthlessness; $33.84 \%$ of the variance) and uniquely identified by infrequency of endorsement (bad person, unloved, anhedonia; $36.92 \%$ of the variance) as predictors of life satisfaction ( $95 \% \mathrm{Cl}$ for $\mathrm{R}^{2}$ pred difference between the models: $-0.01,0.07)$. Therefore, central symptoms predicted significantly more out of fold variance than peripheral symptoms and commonly endorsed symptoms. However, the most central symptoms do not predict more out of fold variance in life satisfaction than the most infrequently endorsed symptoms. This pattern of results holds when using continuous symptom severity as predictors rather than binarized symptom endorsement (See Section S5). 


\section{Discussion}

In the current study, we found that both strength centrality and infrequency of symptom endorsement could identify symptoms that were more strongly associated with life satisfaction. Sadness, self-hatred, loneliness, and worthlessness were the most central symptoms in adolescent depression while anhedonia, self-hatred, bad person, and unloved were the least

frequently endorsed symptoms. Although assessed with a different questionnaire in a sample of adolescents recruited from a different part of the United States, the central symptoms identified in this study strongly converge with the central symptoms identified in prior work (Mullarkey et al., 2018). Further, Bayesian replicability estimates using split-halves of the current sample indicated we would expect this adolescent depression network to generally replicate in a new, similar sample.

In line with previous work on adult depression (Fried \& Nesse, 2014), some depression symptoms share far more variance with life satisfaction than others in adolescents. Nearly half of all possible pairwise comparisons revealed one symptom of depression sharing significantly more variance with life satisfaction than another symptom. Further, the variance depression symptoms shared with life satisfaction ranged from $2.9 \%$ (feeling restless) to $12.6 \%$ (sadness). These results provide the first empirical evidence that some symptoms of depression are more closely associated with life satisfaction than others in adolescents. Sadness in adults also shares the most variance out of any depression symptom with psychosocial functioning (Fried \& Nesse, 2014), so future developmental psychopathology research should examine whether sadness is the strongest predictor of other important outcomes.

Importantly, strength centrality and infrequency of endorsement identified which symptoms were more strongly related to life satisfaction. Applying a bootstrapping method to the estimation of the network and calculating variance each symptom shares with life satisfaction allowed us to calculate more robustly whether strength centrality and infrequency of endorsement are significantly associated with the variance a symptom shares with life 
satisfaction. Our second method included out of fold prediction, implying the more central symptoms alone could predict $35.55 \%$ of the variance in adolescent life satisfaction in unseen data while infrequently endorsed samples could predict $37.30 \%$. In contrast, the least central, or peripheral symptoms, could only predict $29.22 \%$ of the out of fold variance in life satisfaction, while commonly endorsed symptoms could only predict $26.84 \%$ - both significantly less than the most central symptoms.

Symptom centrality and symptom endorsement rate were modestly negatively associated in this sample, and thus are likely not redundant. Further, our analyses indicate strength centrality was still positively associated with how much variance a symptom shared with life satisfaction after controlling for endorsement rate. While endorsement rate technically no longer significantly relates to how much variance a symptom shares with life satisfaction after controlling for centrality, the impact of the covariate was relatively small. Symptoms identified uniquely by infrequency of endorsement (anhedonia, bad person, unloved) predict a similar amount of out-of-fold variance in life satisfaction as symptoms uniquely identified by centrality (sadness, loneliness, worthlessness).

Symptom centrality and infrequency of symptom endorsement may both be viable ways to identify more important symptoms within adolescent depression. Infrequency of endorsement may be an especially useful symptom level metric in samples where identifying central symptoms via network analysis is not appropriate (e.g., the sample size is too small to estimate a network where strength is stable). Empirically testing different methods for identifying more important symptoms of adolescent depression across a variety of outcomes will help us develop a more nuanced picture of which frameworks for identifying important symptoms will be practically useful.

An important limitation of these analyses is the cross-sectional nature of the data. Variance shared is not variance predicted over time, much less a causal relationship, and any interpretations drawn from these data should be tempered by those caveats. Another potential 
limitation of these analyses is that they were drawn from community adolescents, rather than a clinical population. Recruiting a clinical sample could change the relationship between individual symptoms and life satisfaction. Although past work demonstrates that network structure does not differ by diagnostic status, it could influence the overall connectivity of the network (Santos et al., 2017). However, there are advantages to using a sample not selected for clinical severity, as none of the statistical procedures can be confounded by Berkson's or collider bias - where selecting on one variable (e.g, clinical severity) distorts the associations between other variables of interest (de Ron et al., 2019). Selecting for clinical severity will also inherently restrict the range of which symptoms are more vs. less frequently endorsed. Future research could examine if infrequency of endorsement still identifies informative symptoms for outcomes despite this range restriction in clinical samples.

Overall, these results indicate that the most central symptoms of adolescent depression include self-hatred, worthlessness, loneliness, and sadness while the least frequently endorsed were self-hatred, anhedonia, feeling like a bad person, and feeling unloved. Importantly, the central symptoms were consistent with prior work even though they were identified with a different measure of depression. Further, depression symptom centrality and infrequency of endorsement were strongly associated with life satisfaction across methods whereas a higher endorsement rate was not. This finding further supports the informativeness of the network approach (i.e., external validity) for identifying central symptoms of adolescent depression, though in this case infrequency of symptom endorsement was just as informative. Longitudinal studies are needed to ascertain if individual depression symptoms differentially predict life satisfaction over time, and also whether life satisfaction reciprocally predicts change in specific symptoms. Intervention studies that target central and infrequently endorsed depression symptoms could also help disentangle whether the relationships between individual depression symptoms and life satisfaction are causal in nature. Nevertheless, findings from the current study suggest that self-hatred, worthlessness, loneliness, sadness, feeling unloved, and feeling 
like a bad person may be particularly pertinent to the experience of depression in adolescence especially in how adolescent depression relates to concurrent life satisfaction. Applying symptom level approaches to adolescent depression may provide new theoretical and clinical insights that further our understanding of this heterogeneous disorder. 


\section{References}

Beevers, C. G., Mullarkey, M. C., Dainer-Best, J., Stewart, R. A., Labrada, J., Allen, J. J. B., McGeary, J. E., \& Shumake, J. (2018). Association between negative cognitive bias and depression: A symptom-level approach. In PsyArXiv. https://doi.org/10.31234/osf.io/d3xfh

Bertha, E. A., \& Balázs, J. (2013). Subthreshold depression in adolescence: a systematic review. European Child \& Adolescent Psychiatry, 22(10), 589-603.

Bluth, K., Campo, R. A., Futch, W. S., \& Gaylord, S. A. (2017). Age and Gender Differences in the Associations of Self-Compassion and Emotional Well-being in A Large Adolescent Sample. Journal of Youth and Adolescence, 46(4), 840-853.

Bluth, K., Mullarkey, M., \& Lathren, C. (2018). Self-Compassion: A Potential Path to Adolescent Resilience and Positive Exploration. Journal of Child and Family Studies, 27(9), 30373047.

Borsboom, D. (2017). A network theory of mental disorders. World Psychiatry: Official Journal of the World Psychiatric Association , 16(1), 5-13.

Borsboom, D., Fried, E. I., Epskamp, S., Waldorp, L. J., van Borkulo, C. D., van der Maas, H. L. J., \& Cramer, A. O. J. (2017). False alarm? A comprehensive reanalysis of "Evidence that psychopathology symptom networks have limited replicability" by Forbes, Wright, Markon, and Krueger (2017). Journal of Abnormal Psychology, 126(7), 989-999.

Boschloo, L., van Borkulo, C. D., Borsboom, D., \& Schoevers, R. A. (2016). A Prospective Study on How Symptoms in a Network Predict the Onset of Depression. Psychotherapy and Psychosomatics, 85(3), 183-184.

Bringmann, L., Elmer, T., \& Snippe, E. (2018). What do centrality measures measure in psychological networks?

Crowe, E., Daly, M., Delaney, L., Carroll, S., \& Malone, K. M. (2018). The intra-day dynamics of affect, self-esteem, tiredness, and suicidality in Major Depression. Psychiatry Research. 
https://doi.org/10.1016/j.psychres.2018.02.032

de Ron, J., Fried, E. I., \& Epskamp, S. (2019). Psychological Networks in Clinical Populations: A tutorial on the consequences of Berkson's Bias. In PsyArXiv.

Epskamp, S., Borsboom, D., \& Fried, E. I. (2017). Estimating psychological networks and their accuracy: a tutorial paper. Behavior Research Methods, 1-18.

Epskamp, S., \& Fried, E. I. (2016). A Tutorial on Regularized Partial Correlation Networks. In arXiv [stat.AP]. arXiv. http://arxiv.org/abs/1607.01367

Fried, E. I. (2015). Problematic assumptions have slowed down depression research: why symptoms, not syndromes are the way forward. Frontiers in Psychology, 6, 1-11.

Fried, E. I. (2017). The 52 symptoms of major depression: Lack of content overlap among seven common depression scales. Journal of Affective Disorders, 208, 191-197.

Fried, E. I., Bockting, C., Arjadi, R., Borsboom, D., Amshoff, M., Cramer, A. O. J., Epskamp, S., Tuerlinckx, F., Carr, D., \& Stroebe, M. (2015). From loss to loneliness: The relationship between bereavement and depressive symptoms. Journal of Abnormal Psychology, 124(2), 256-265.

Fried, E. I., Eidhof, M. B., Palic, S., Costantini, G., Huisman-van Dijk, H. M., Bockting, C. L. H., Engelhard, I., Armour, C., Nielsen, A. B. S., \& Karstoft, K.-I. (2018). Replicability and Generalizability of Posttraumatic Stress Disorder (PTSD) Networks: A Cross-Cultural Multisite Study of PTSD Symptoms in Four Trauma Patient Samples. Clinical Psychological Science, 6(3), 335-351.

Fried, E. I., \& Nesse, R. M. (2014). The Impact of Individual Depressive Symptoms on Impairment of Psychosocial Functioning. PloS One, 9(2), e90311.

Fried, E. I., \& Nesse, R. M. (2015). Depression is not a consistent syndrome: an investigation of unique symptom patterns in the STAR*D study. Journal of Affective Disorders, 172, 96102.

Gabbay, V., Johnson, A. R., Alonso, C. M., Evans, L. K., Babb, J. S., \& Klein, R. G. (2015). 
Anhedonia, but not Irritability, Is Associated with Illness Severity Outcomes in Adolescent Major Depression. Journal of Child and Adolescent Psychopharmacology, 25(3), 194-200.

Gadermann, A. M., Guhn, M., Schonert-Reichl, K. A., Hymel, S., Thomson, K., \& Hertzman, C. (2016). A population-based study of children's well-being and health: The relative importance of social relationships, health-related activities, and income. Journal of Happiness Studies, 17(5), 1847-1872.

Hauser, D. J., \& Schwarz, N. (2016). Attentive Turkers: MTurk participants perform better on online attention checks than do subject pool participants. Behavior Research Methods, 48(1), 400-407.

Huebner, E. S. (1991). Initial development of the student's life satisfaction scale. School Psychology International, 12(3), 231-240.

Huebner, E. S., Funk, B. A., III, \& Gilman, R. (2000). Cross-sectional and longitudinal psychosocial correlates of adolescent life satisfaction reports. Canadian Journal of School Psychology, 16(1), 53-64.

Jones, P. J., Williams, D. R., \& McNally, R. J. (2020). Sampling Variability Is Not Nonreplication: A Bayesian Reanalysis of Forbes, Wright, Markon, and Krueger. In Multivariate Behavioral Research (pp. 1-7). https://doi.org/10.1080/00273171.2020.1797460

Kuo, E. S., Stoep, A. V., \& Stewart, D. G. (2005). Using the short mood and feelings questionnaire to detect depression in detained adolescents. Assessment, 12(4), 374-383. Lu, W. (2019). Adolescent Depression: National Trends, Risk Factors, and Healthcare Disparities. American Journal of Health Behavior, 43(1), 181-194.

Marchetti, I., Everaert, J., Dainer-Best, J., Loeys, T., Beevers, C. G., \& Koster, E. H. W. (2018). Specificity and overlap of attention and memory biases in depression. Journal of Affective Disorders, 225, 404-412.

Marchetti, I., Loeys, T., Alloy, L. B., \& Koster, E. H. W. (2016). Unveiling the Structure of Cognitive Vulnerability for Depression: Specificity and Overlap. PloS One, 11(12), 
e0168612.

Marsman, M., \& Wagenmakers, E.-J. (2017). Bayesian benefits with JASP. European Journal of Developmental Psychology, 14(5), 545-555.

McCuish, E., Bouchard, M., Beauregard, E., \& Corrado, R. (2019). A Network Approach to Understanding the Structure of Core Symptoms of Psychopathic Personality Disturbance in Adolescent Offenders. Journal of Abnormal Child Psychology.

https://doi.org/10.1007/s10802-019-00530-9

McKenzie, D. P., Toumbourou, J. W., Forbes, A. B., Mackinnon, A. J., McMorris, B. J., Catalano, R. F., \& Patton, G. C. (2011). Predicting future depression in adolescents using the Short Mood and Feelings Questionnaire: A two-nation study. Journal of Affective Disorders, 134(1), 151-159.

McKnight, P. E., \& Kashdan, T. B. (2009). The importance of functional impairment to mental health outcomes: a case for reassessing our goals in depression treatment research. Clinical Psychology Review, 29(3), 243-259.

McNally, R. J. (2016). Can network analysis transform psychopathology? Behaviour Research and Therapy, 86, 95-104.

Mullarkey, M. C., Marchetti, I., \& Beevers, C. G. (2018). Using Network Analysis to Identify Central Symptoms of Adolescent Depression. Journal of Clinical Child and Adolescent Psychology: The Official Journal for the Society of Clinical Child and Adolescent Psychology, American Psychological Association, Division 53.

https://doi.org/10.1080/15374416.2018.1437735

Mullarkey, M. C., Stewart, R. A., Wells, T. T., Shumake, J., \& Beevers, C. G. (2019). Self-Dislike and Sadness are Central Symptoms of Depression in College Students: A Network Analysis. https://doi.org/10.31234/osf.io/fujmb

Proctor, C. L., Linley, P. A., \& Maltby, J. (2009). Youth Life Satisfaction: A Review of the Literature. Journal of Happiness Studies, 10(5), 583-630. 
Rodebaugh, T. L., Tonge, N. A., Piccirillo, M. L., Fried, E., Horenstein, A., Morrison, A. S., Goldin, P., Gross, J. J., Lim, M. H., Fernandez, K. C., Blanco, C., Schneier, F. R., Bogdan, R., Thompson, R. J., \& Heimberg, R. G. (2018). Does centrality in a cross-sectional network suggest intervention targets for social anxiety disorder? Journal of Consulting and Clinical Psychology, 86(10), 831-844.

Santos, H., Fried, E. I., Asafu-Adjei, J., \& Ruiz, R. J. (2017). Network structure of perinatal depressive symptoms in Latinas: relationship to stress and reproductive biomarkers. Research in Nursing \& Health, 40(3), 218-228.

Schimmack, U. (2008). The structure of subjective well-being. The Science of Subjective WellBeing, 97-123.

Terluin, B., de Boer, M. R., \& de Vet, H. C. W. (2016). Differences in Connection Strength between Mental Symptoms Might Be Explained by Differences in Variance: Reanalysis of Network Data Did Not Confirm Staging. PloS One, 11(11), e0155205.

Thapar, A., \& McGuffin, P. (1998). Validity of the shortened Mood and Feelings Questionnaire in a community sample of children and adolescents: a preliminary research note. Psychiatry Research, 81(2), 259-268.

Valente, T. W. (2012). Network interventions. Science, 337(6090), 49-53.

van Borkulo, C. D., Borsboom, D., Epskamp, S., Blanken, T. F., Boschloo, L., Schoevers, R. A., \& Waldorp, L. J. (2014). A new method for constructing networks from binary data. Scientific Reports, 4(5918). https://doi.org/10.1038/srep05918 Vazire, S. (2018). Implications of the Credibility Revolution for Productivity, Creativity, and Progress. PsyArXiv. https://doi.org/10.17605/OSF.IO/2YPHF

Williams, D. R., Rast, P., Pericchi, L. R., \& Mulder, J. (2020). Comparing Gaussian graphical models with the posterior predictive distribution and Bayesian model selection.

Psychological Methods. https://doi.org/10.1037/met0000254

Zadow, C., Houghton, S., Hunter, S. C., Rosenberg, M., \& Wood, L. (2017). Associations 
between positive mental well-being and depressive symptoms in Australian adolescents.

The Educational and Developmental Psychologist, 34(2), 95-105. 


\title{
Supplementary Material for Symptom centrality and infrequency of endorsement identify adolescent depression symptoms more strongly associated with life satisfaction
}

\author{
Michael Mullarkey
}

September 07, 2020

\section{Section S1: A Brief Explainer of Cross Validation}

K-fold cross-validation is a procedure similar to creating testing and training datasets in machine learning. Sample sizes typical in psychology, however, do not easily allow for holding out a portion of the data to be "tested" after a predictive model has been "trained" on an initial part of the data. Without cross-validation, however, we tend to overestimate how much our models will apply to out of sample data.

One way to solve this predicament is K-Fold cross-validation. If we have (for example) 10 folds, we divide the data into 10 random sections and train the model on the first 9 . We then see how much variance that model predicts in the 10 th section (test set). We then repeat this process 10 times and average how much variance the model predicts across the 10 test sets.

We can then use the "compare" function from the beset package in R to determine which models (e.g., a model with central symptoms vs. a model with peripheral symptoms) predict the most cross-validated variance in an outcome (e.g., well-being).

In other words, these analyses give us a way to directly compare the predictive ability of symptoms expected to be important under network, severity, and endorsement models head to head.

Using these analyses also allows us to use all of our substantial sample size $(1,055)$ rather than relying on correlations between statistics like mean and symptom strength $(n=13$, or number of symptoms).

Section S2: Item Informativenes 
table(dataset\$mfq2)/10.59 \# as for the item with the lowest variance, only 66 $\%$ report no symptom, whereas $34 \%$ report the presence of the symptom

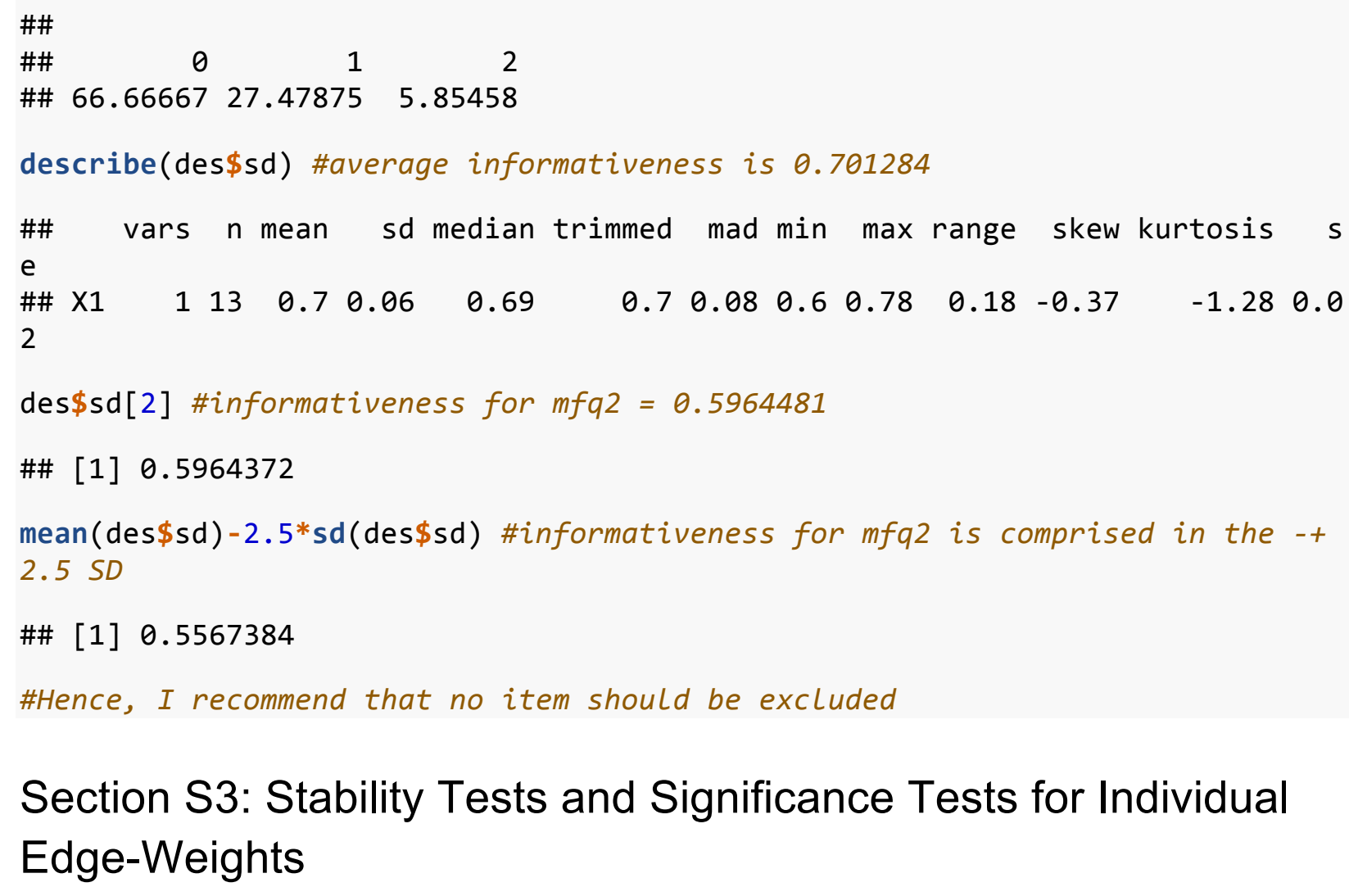

\section{Section S3: Stability Tests and Significance Tests for Individual Edge-Weights}

\#\# Re-estimate to have proper labesls for the figures

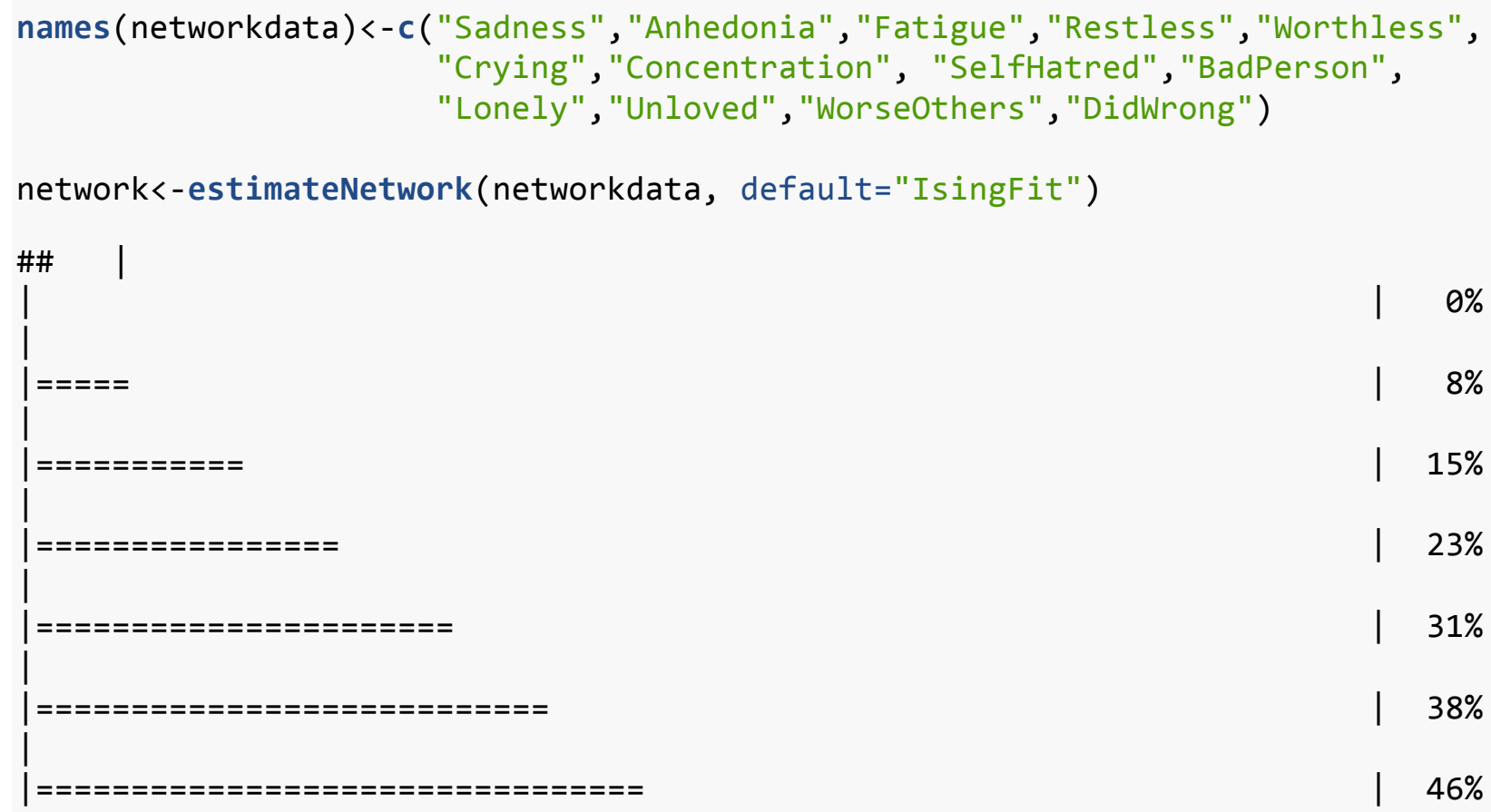




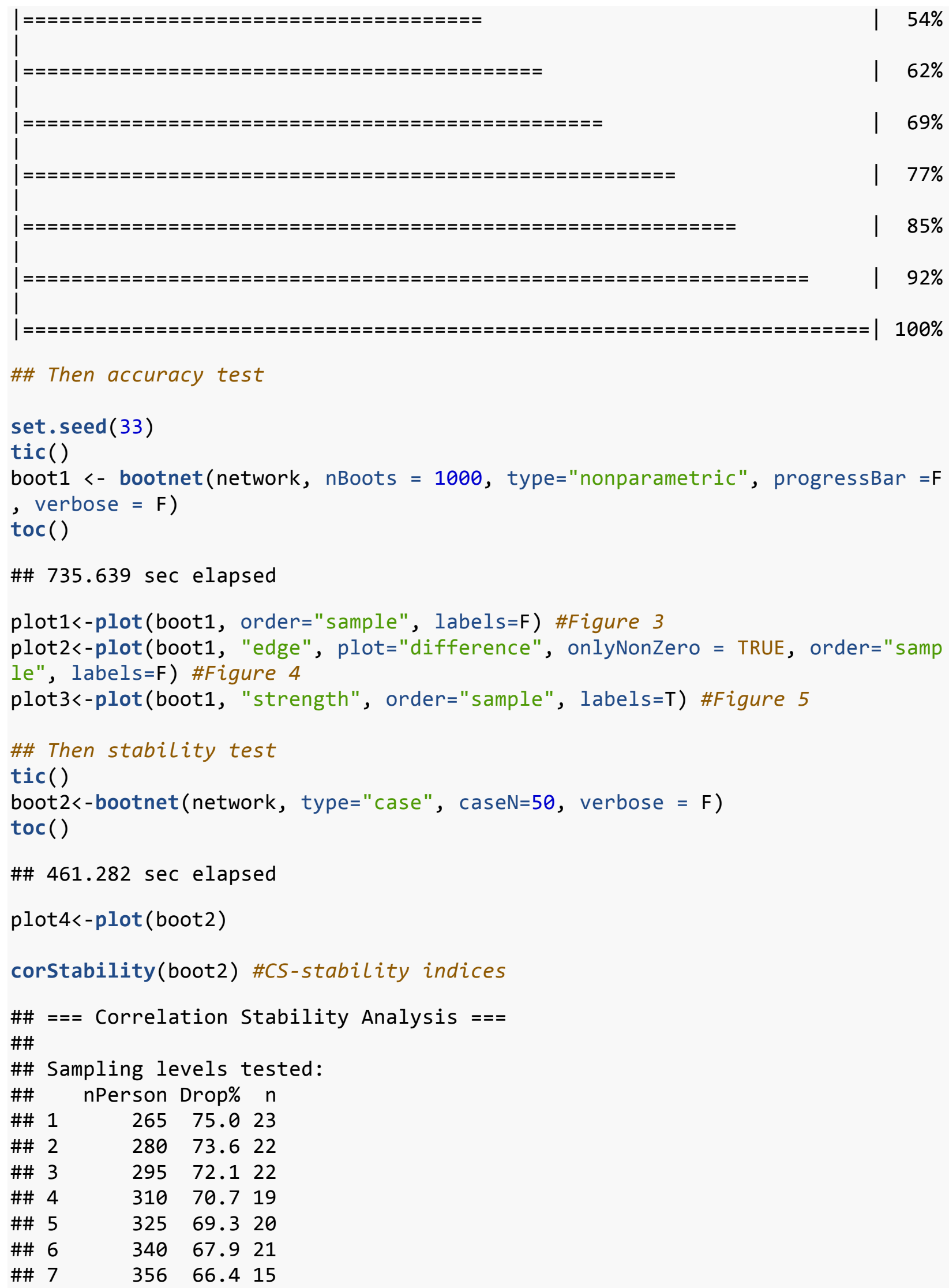




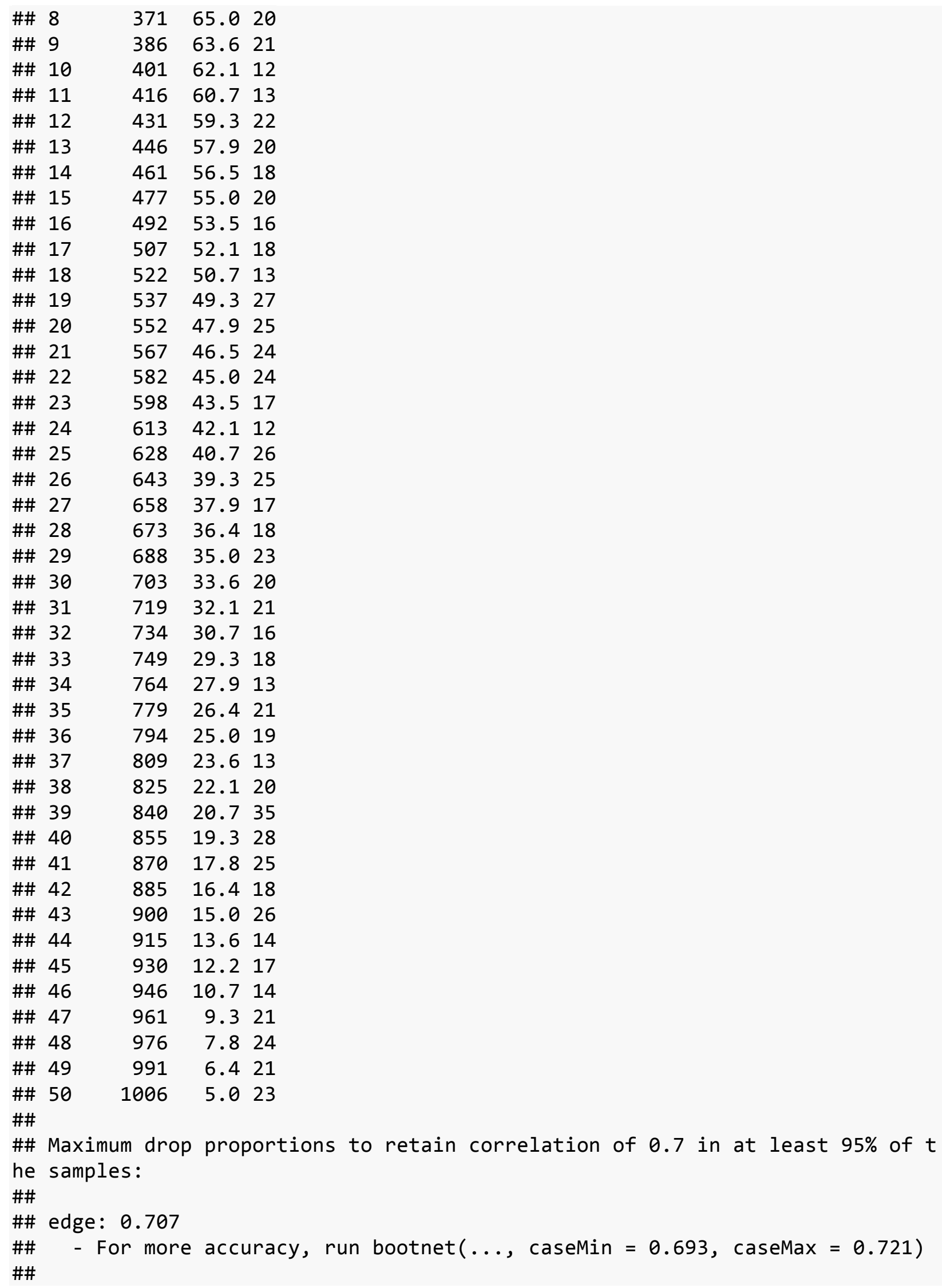


\#\# intercept: 0.75 (CS-coefficient is highest level tested)

\#\# - For more accuracy, run bootnet $(. . .$, caseMin $=0.736$, caseMax $=1$ )

\#\#

\#\# strength: 0.75 (CS-coefficient is highest level tested)

\#\# - For more accuracy, run bootnet $(. .$, caseMin $=0.736$, caseMax $=1$ )

\#\#

\#\# Accuracy can also be increased by increasing both 'nBoots' and 'caseN'.

plot2 \# Significance tests for individual edge-weights

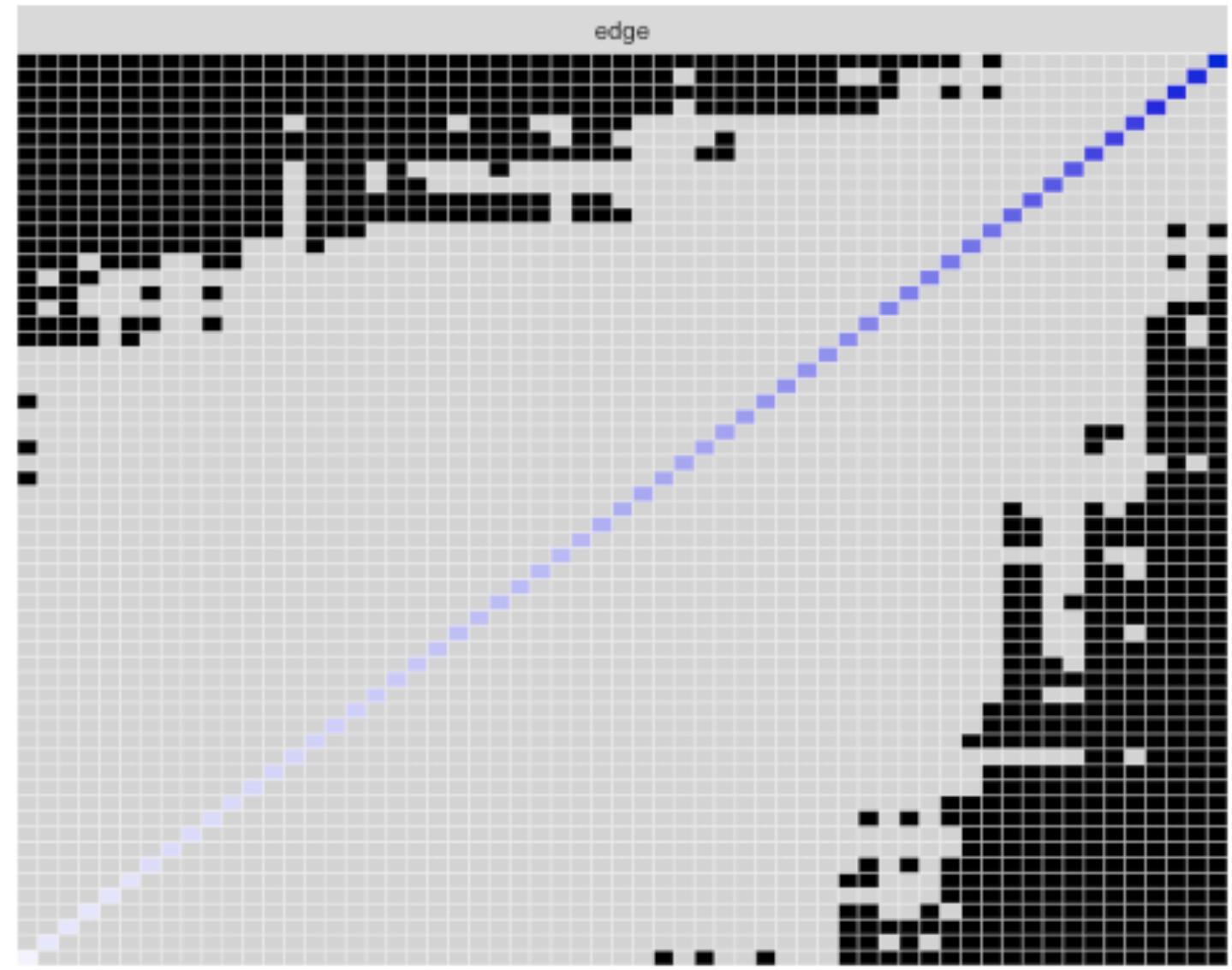

Section S4: Associations Between Strength Centrality, Endorsement Frequency, and Standard Deviation of Symptoms

\section{Bootstrapped Correlation Between Centrality and SD of Items}

\#\# One-time correlation: $r=-0.1195 \%$ CI $(-0.35,0.08)$

ad_dep_well_being_centrality_sd_binarized<-cor(psych: :describe(dataset_binari zed $[, 2: 14]) \$ s d$, CentrIndex_Study_2\$node.centrality\$Strength)

ad_dep_well_being_centrality_sd_binarized 


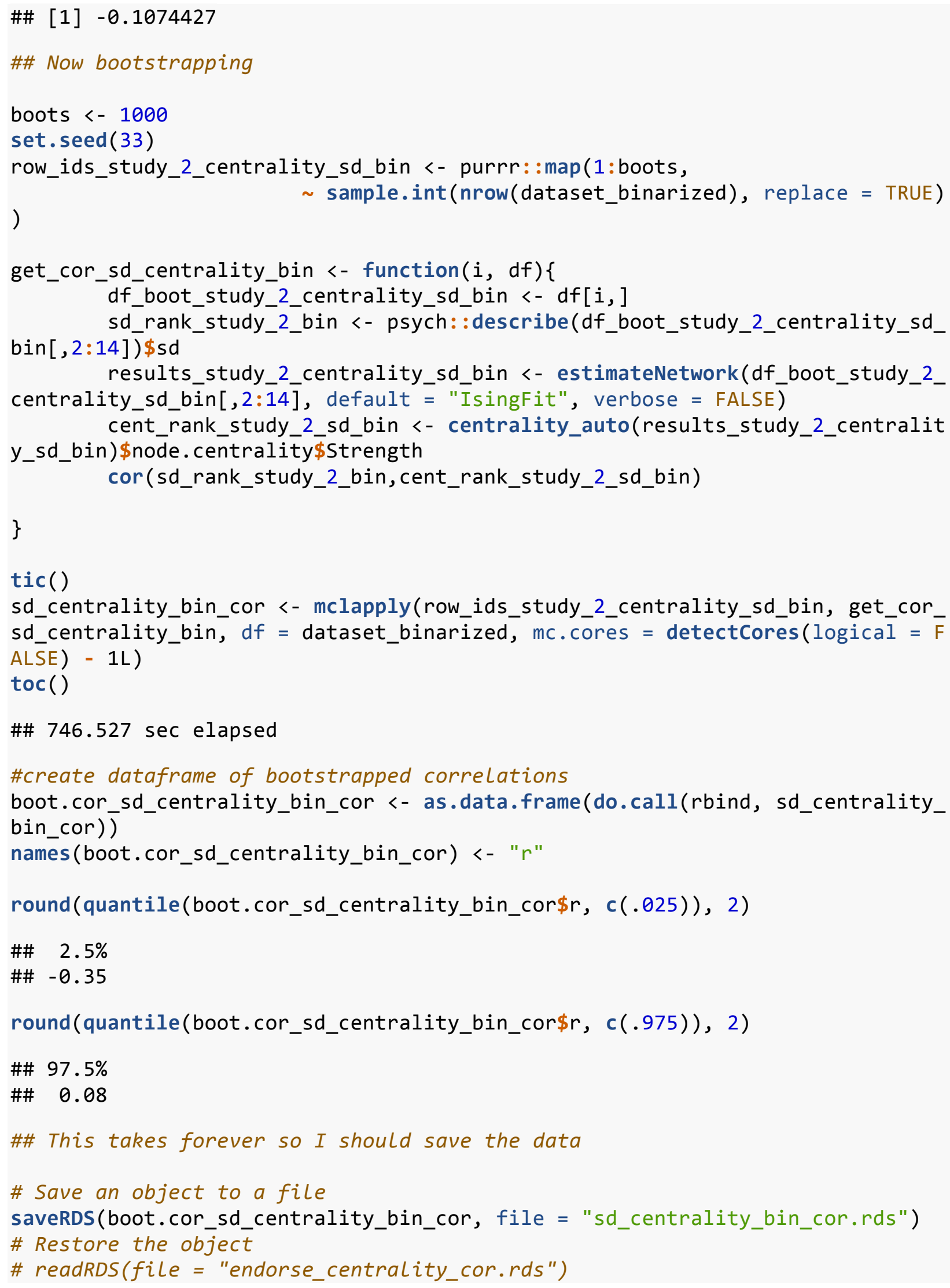




\section{Bootstrapped Correlation Between Centrality and Endorsement Frequency of Items}

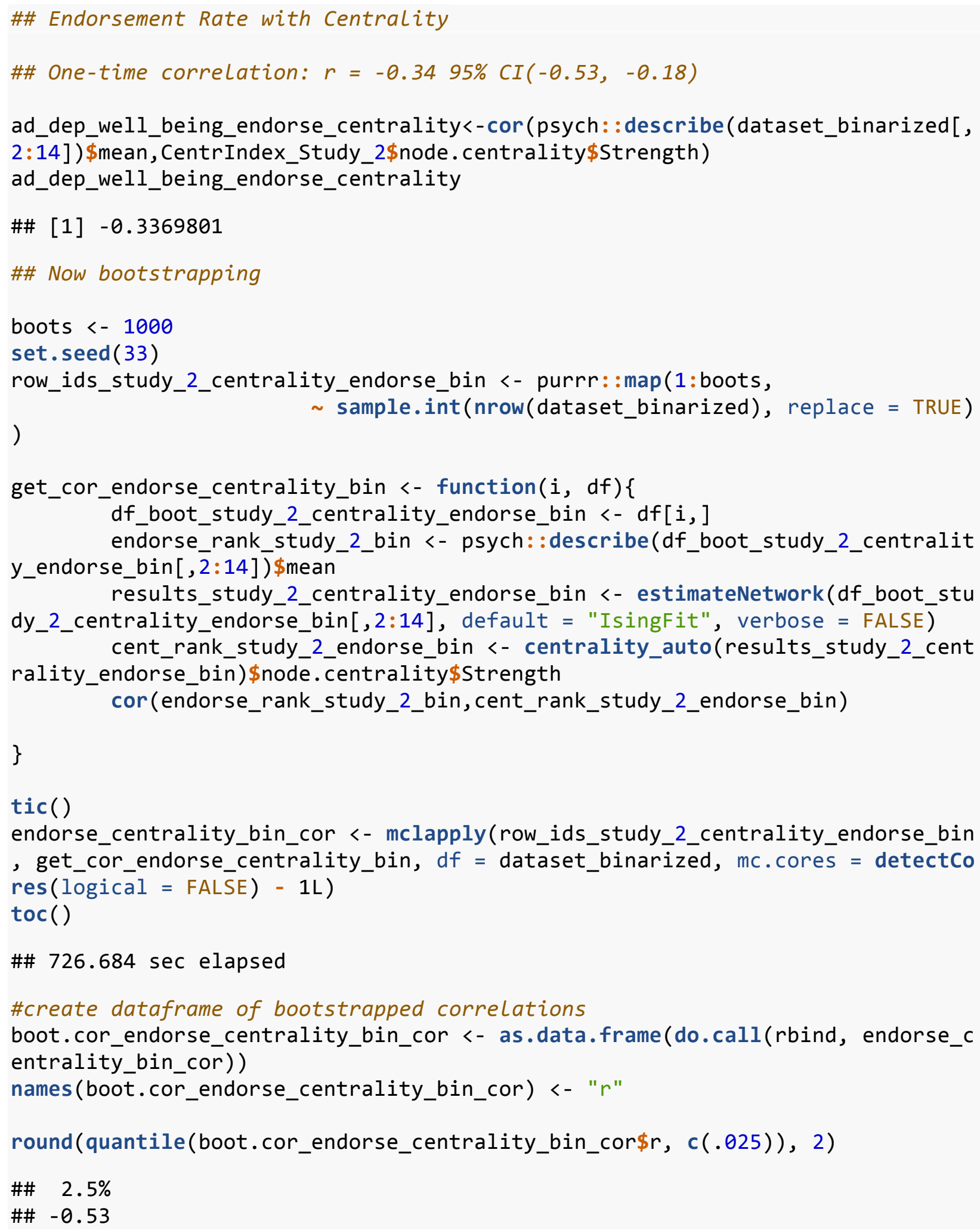




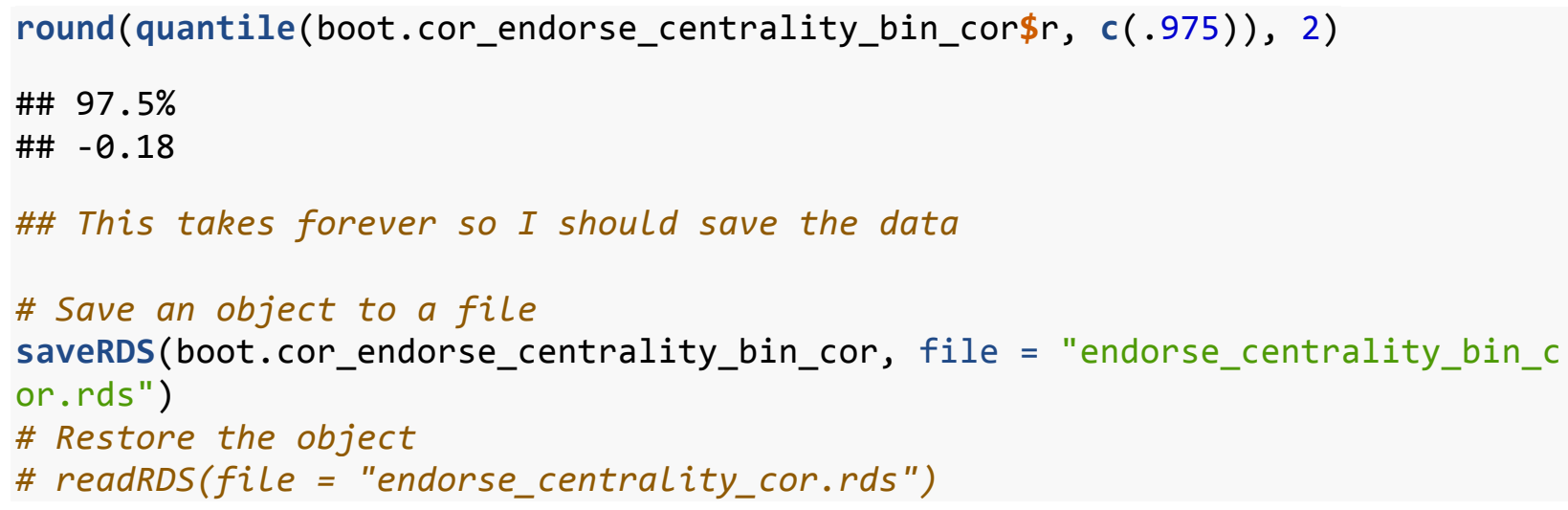

\section{Section S5: Continuous Symptoms as Predictors of Life Satisfaction in Cross-Validated Linear Regressions}

\section{Defining Central, Peripheral, Severe, and Endorsement}

We selected the most central symptoms by seeing which symptoms were stronger than $50 \%$ or more of the other symptoms in the network (sadness, self-hatred, loneliness, and worthlessness).

For peripheral symptoms, we chose the 4 least central symptoms (to match the number of central symptoms) indexed by strength in this network (fatigue, restless, crying, and anhedonia).

\section{Identifying Most and Least "Severe" Symptoms}

For the most severe symptoms, we chose the 4 symptoms with the highest mean ratings on severity from our continuous data (fatigue, restless, concentration, and sadness).

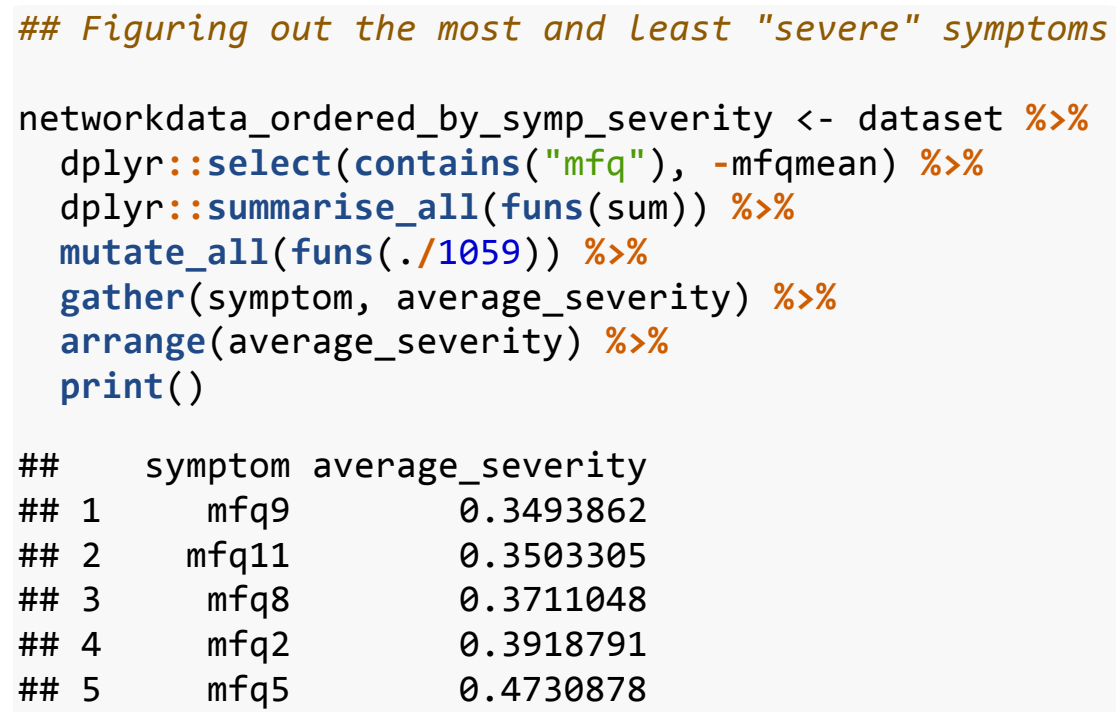




\begin{tabular}{|c|c|c|c|}
\hline \#\# & 6 & $\mathrm{mfq} 13$ & 0.4995279 \\
\hline \#\# & 7 & $m f q 6$ & 0.5297450 \\
\hline$\# \#$ & 8 & $\mathrm{mfq} 12$ & 0.5580737 \\
\hline$\# \# \subseteq$ & 9 & mfq10 & 0.6270066 \\
\hline \#\# 1 & 10 & $m f q 1$ & 0.7837583 \\
\hline \#\# 1 & 11 & $\mathrm{mfq4}$ & 0.8016997 \\
\hline \#\# 1 & 12 & $\mathrm{mfq} 7$ & 0.8470255 \\
\hline \#\# 1 & 13 & $m f q 3$ & 0.915958 \\
\hline
\end{tabular}

\section{The Most Central Symptoms Predict More Variance in Well-Being Than the Least Central Symptoms}

A model with the most central symptoms predicts $39.02 \%$ of the variance in well-being, while a model with the most peripheral symptoms predicts $29.31 \%$. When comparing the models, the $95 \%$ CI for the difference between them does not include $0(-0.14,-0.06)$.

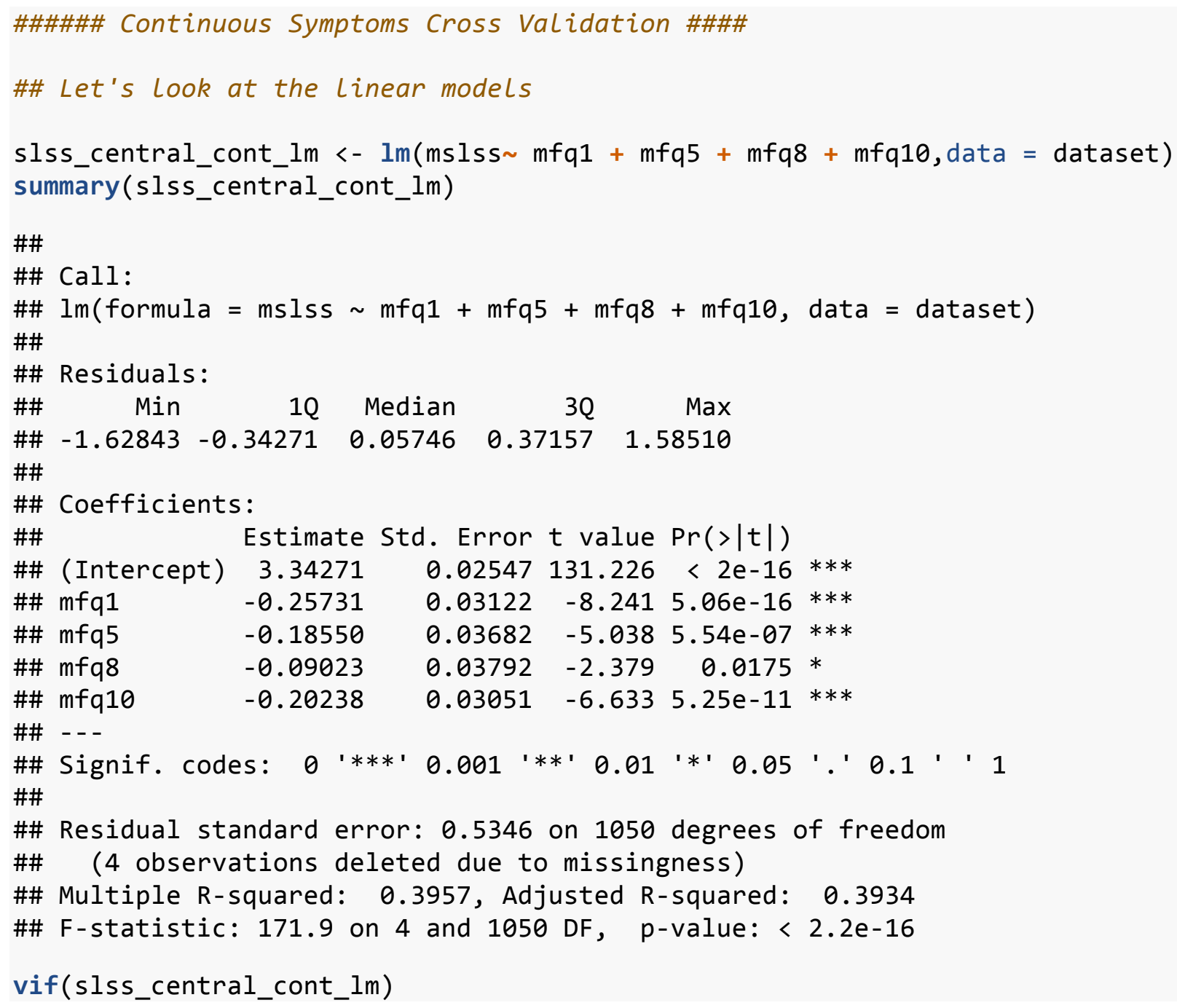




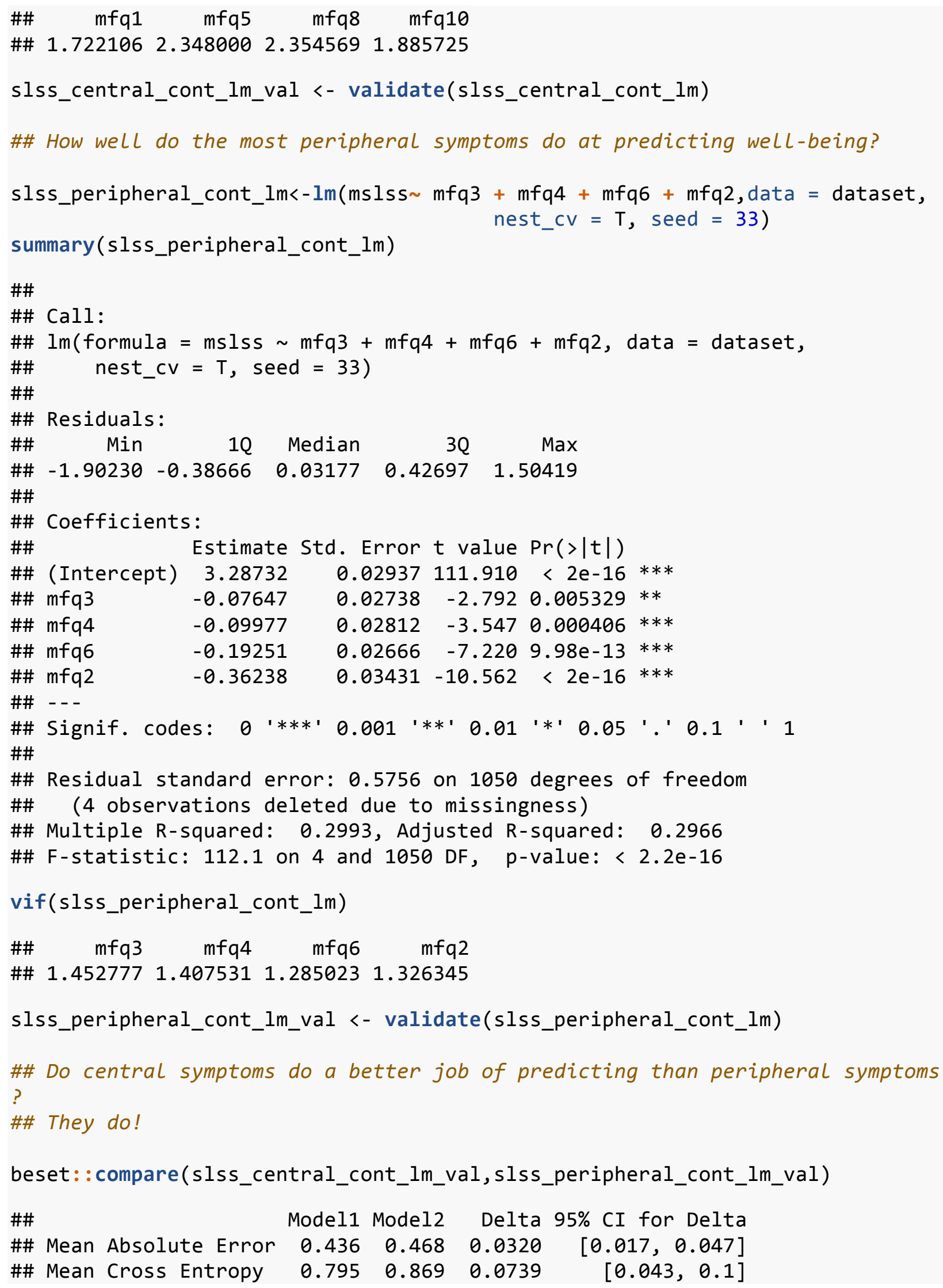


\#\# Mean Squared Error

$\left.\begin{array}{llll}0.287 & 0.333 & 0.0457 \quad[0.026,0.065\end{array}\right]$

\#\# Variance Explained

$0.390 \quad 0.293-0.0971 \quad[-0.14,-0.055]$

\section{The Most Central Symptoms Predict More Variance in Well-Being Than the "Most Severe" On Average Symptoms}

A model with the most central symptoms predicts $39.02 \%$ of the variance in well-being, while a model with the most severe symptoms predicts $32.58 \%$. When comparing the models, the $95 \%$ CI for the difference between them does not include $0(-0.09,-0.03)$.

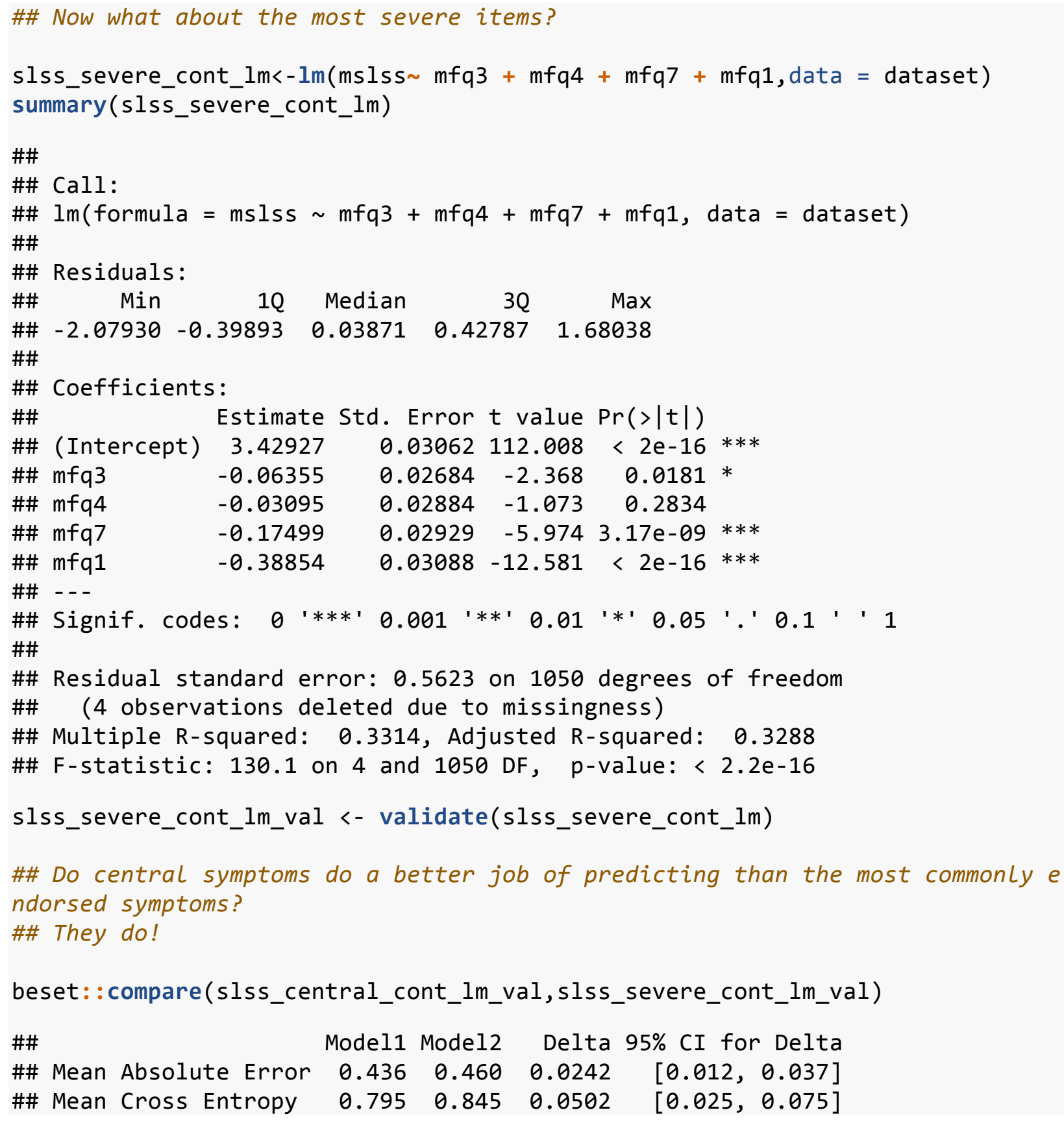


\#\# Mean Squared Error

$\left.\begin{array}{lllll}0.287 & 0.317 & 0.0303 \quad[0.015,0.045\end{array}\right]$

\#\# Variance Explained

0.390

$0.326-0.0644[-0.096,-0.032]$

\section{The Most Central Symptoms Do Not Predict More Variance in Well-Being Than the "Least Severe" On Average Symptoms}

A model with the most central symptoms predicts $39.02 \%$ of the variance in well-being, while a model with the least severe symptoms predicts $35.71 \%$. When comparing the models, the $95 \%$ CI for the difference between them does not include $0(-0.07,002)$.

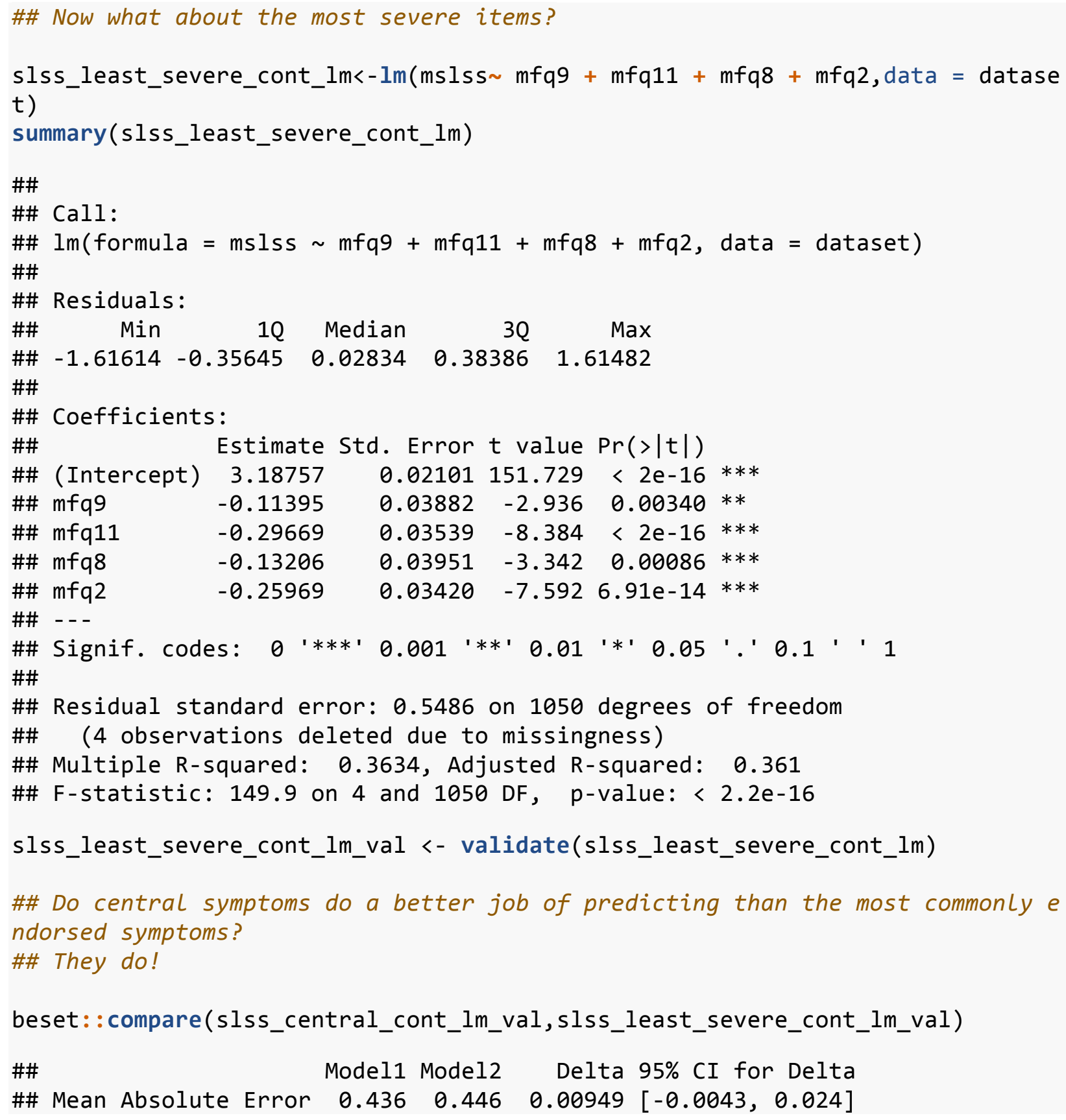


\#\# Mean Cross Entropy \#\# Mean Squared Error \#\# Variance Explained
$0.795 \quad 0.8210 .02637 \quad[-0.0015,0.056]$

$\begin{array}{llllll}0.287 & 0.303 & 0.01554 & {[-9 \mathrm{e}-04,0.033]}\end{array}$

$\begin{array}{lllll}0.390 & 0.357 & -0.03303 & {[-0.07,0.002]}\end{array}$ 